\title{
Behaviour of the Germ Cell Specific Lamin Through Mammalian Spermatogenesis as Probed with Monoclonal Antibodies*
}

\author{
K. Manjula, Anjali Karande and M.R.S. Rao ${ }^{\dagger 1}$ \\ Department of Biochemistry, $\dagger$ Jawaharlal Nehru Centre for Advanced Study, Indian Institute of Science, \\ Bangalore 560012, INDIA
}

Key words: germ cell specific lamin/spermatogenesis

\begin{abstract}
$A B S T R A C T$. We had earlier identified a $60 \mathrm{kDa}$ nuclear lamin protein (lamin $\left.{ }_{g}\right)$ unique to the germ cells of rat testis which was subsequently shown to be antigenically conserved in germ cells of grasshopper, rooster, frog

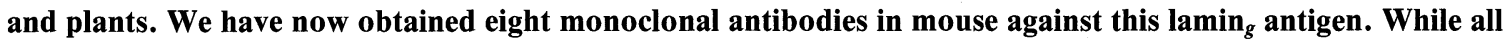
the eight Mabs reacted with lamin $g$ antigen in an immunoblot analysis, only three $\mathrm{Mabs}\left(A_{11} C_{7}, A_{11} D_{4}, C_{1} F_{7}\right)$ showed strong reactivity in the immunofluorescence analysis of the germ cells. The Mabs $A_{11} C_{7}$ and $A_{11} D_{4}$ showed a slight cross-reactivity with rat liver lamin B. Indirect immunofluorescence analysis of pre-meiotic, mei-

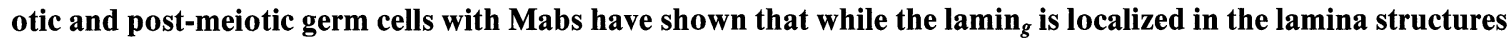
of spermatogonia and round spermatids, it is localized to the phase dense regions of pachytene spermatocytes which is in conformity with our previous observations using rabbit polyclonal antibodies. The localization of the antigen in the germ cells was also confirmed by immunohistochemical staining of the thin sections of seminiferous tubules. By immunostaining the surface spread pachytene spermatocytes, the antigen was further localized to the telomeric ends of the paired homologous chromosomes. Using anti-somatic lamin B antibodies, we have also demonstrated the absence of somatic lamins in meiotic and post-meiotic germ cells. The lamina structure of pre-meiotic spermatogonial nucleus contains both somatic lamin $B$ and lamin $_{g}$ as evidenced by immuno-

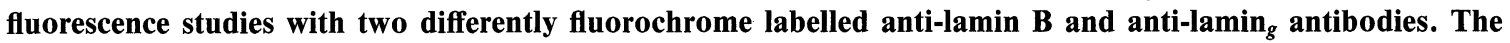
selective retention of $\operatorname{lamin}_{g}$ in the pachytene spermatocytes is probably essential for anchoring the telomeric ends of the paired chromosomes to the inner nuclear membrane.
\end{abstract}

The nuclear lamina is an important ubiquitous proteinaceous structure present in eukaryotic cells subjacent to the inner nuclear membrane (15). In addition to providing mechanical stability to the shape of the nucleus, the lamina structure also provides attachment sites for chromatin in the interphase nucleus. In the vertebrates, this lamina structure is composed of three major polypeptides, namely lamins A, B and $\mathrm{C}$, which have a molecular mass in the range of 60 to 80 kilodaltons. Recently, Kaufmann (13) has reported two more acidic lamins, D and E, in the rat liver. Furthermore, lamin B which was originally thought to be a single polypeptide is now shown to be made up of a major component lamin $B_{1}$ and a minor one lamin $B_{2}(17)$.

The nuclear lamina structure is dynamic in nature. For example, during cell division, at the metaphase, the lamins undergo hyperphosphorylation. Consequently, the lamina structure is depolymerized to yield individual lamina polypeptides. During this process, lamins A

\footnotetext{
* This paper is dedicated to the memory of Dr. L. Sudhakar.

1 To whom Communication to be addressed.
}

and $\mathrm{C}$ have been shown to be present in the cytoplasm, while lamin B is associated with the membrane vesicles (8). The depolymerization of the lamina structure is believed to be essential for the condensation of the chromosomes. Following the mitotic division, the lamina structure is reformed at the telophase interval (7). The disassembly of the lamina structure is also observed at the onset of meiosis (26). Such a disassembly process is again thought to be necessary to facilitate chromosome condensation. By using specific antibodies to somatic lamins, Stick and Schwarz (26) observed that there are no remnants of the lamina structure in the meiotic prophase pachytene nucleus. Furthermore, they could not detect any lamina structure in the haploid round spermatids using the same antibodies. However, we have recently shown the presence of a germ cell specific lamin which is expressed only in the germ cells of various eukaryotic species $(1,27)$. We have termed this lamin as lam$\mathrm{in}_{g}$ and shown it to be retained in the pachytene spermatocyte nucleus even though the lamina structure has disassembled. This $\operatorname{lamin}_{g}$ also becomes the sole component of the lamina structure of the haploid round sper- 
matid nucleus. The rabbit polyclonal antibodies against the $\operatorname{lamin}_{g}$ which was used in most of these studies showed very little cross-reactivity with the somatic lamins A, B and C of rat (28). These observations prompted us to generate specific monoclonal antibodies against lamin ${ }_{g}$ in order to study the epitopes that are exclusively present in $\operatorname{lamin}_{g}$, but not in other somatic lamins. Moreover, these monoclonal antibodies would be valuable tools for studies on the expression of this antigen during the spermatogenic process. We have been successful in generating a set of monoclonal antibodies against $\operatorname{lamin}_{g}$ in mouse and we describe here our studies on the nature and the extent of cross-reactivity these monoclonal antibodies exhibit with somatic lamins. We are also describing the results of the immunolocalization of this antigen in pre-meiotic, meiotic prophase and post-meiotic germ cells using these monoclonal antibodies.

\section{MATERIALS AND METHODS}

Materials. All the biochemicals used, unless specified were from Sigma Chemical Company, St. Louis, MO, U.S.A. FITC labelled goat anti-rabbit IgG, from Cappel Laboratories Inc., Cochranville, PA, U.S.A., HRP-conjugated goat anti-rabbit IgG, rabbit anti-mouse IgG and FITC labelled goat anti-mouse IgG were purchased from Bangalore Genei, Bangalore, India. Nitrocellulose sheets were obtained from Schleicher \& Schuell, Dassel, Germany.

All plasticware used for raising monoclonal antibodies were purchased from Becton and Dickinson Labware. Polyethylene glycol 3500, Nystatin, glutamine, hypoxanthine, aminopterin, thymidine and p-nitrophenyl phosphate were obtained from Sigma Chemical Company, U.S.A. Iscove's modified Dulbecco's medium (IMDM) was purchased from Gibco Laboratories, U.S.A. Fetal calf serum was the product of SERA labs, U.K. Pencillin, streptomycin sulfate and gentamycin were obtained from Alembic Chemicals, Sarabhai Chemicals and Pharmaceutical company of India, Bombay, respectively. All other reagents used were of analytical reagent grade.

Animals. Male albino rats of Wistar strain were used in all the studies. Splenocytes from immunized BALB/c mice were used for establishing hybridoma.

Purification of nuclei. Rats were sacrificed by cervical dislocation, testes removed and decapsulated. Livers were perfused with ice-cold physiological saline before they were excised. The tissues were homogenized in 9 volumes of buffer $\mathrm{A}$ (10 mM Tris- $\mathrm{HCl}$, pH 7.5/25 mM KCl/5 mM $\mathrm{MgCl}_{2} / 1 \mathrm{mM}$ $\mathrm{CaCl}_{2}$ ) containing $0.34 \mathrm{M}$ sucrose in a Teflon Potter-Elvejhem homogenizer. Testicular tissue was homogenized by hand while livers were homogenized with a motor-driven pestle. $\mathrm{Nu}$ clei were isolated from the homogenate as described by Rao et al. (22).

Preparation of sonication-resistant spermatid nuclei. Sonication-resistant nuclei from rat testes represent nuclei from elongating and elongated spermatids. They were isolated from the testes of 60 day old rats as described by Singh and Rao (24). Briefly, testes were homogenized in buffer B (10 mM Tris- $\mathrm{HCl}, \mathrm{pH} 7.4 / 0.1 \mathrm{mM}$ phenyl methyl sulfonyl fluoride $10.1 \%$ Triton $\mathrm{X}-100$ ) containing $0.34 \mathrm{M}$ sucrose, filtered through cheese cloth and centrifuged at $1,000 \times \mathrm{g}$ for $10 \mathrm{~min}$ utes. The pellet was suspended in buffer $B$ and sonicated for 12 bursts at setting 6 for 15 seconds (each duration) with 45 seconds intervals in a Branson (Model B-30) sonifier. The sonicate was then layered on a $10 \mathrm{ml}$ cushion of $1.5 \mathrm{M}$ sucrose in buffer $\mathrm{B}$ and centrifuged at $1,000 \times \mathrm{g}$ for 30 minutes. The purity of the pelleted sonication resistant nuclei was checked under a microscope.

Preparation of liver nuclear lamina and the DNase 1 and high salt resistant fraction of sonication resistant nuclei. $\mathrm{Nu}-$ clear lamina fraction was isolated from purified rat liver nuclei essentially according to the method of Dwyer and Blobel (5). The sonication resistant nuclei of rat testes were also subjected to a similar procedure as described by Sudhakar et al. (28) to obtain in DNase 1 and high salt resistant fraction.

$S D S-P A G E$, Electroelution and Immunoblotting. Analysis of the polypeptides was done on SDS 5-15\% gradient polyacrylamide gels essentially according to the method of Laemmli (16). The polypeptides were visualized by staining with $0.1 \%$ coomassie brilliant blue. In some cases, the gel was stained with $\mathrm{AgNO}_{3}$ as described by Merril et al. (19).

For electroelution of $\operatorname{lamin}_{g}$, the polypeptides of the sonication resistant nuclei were separated on a gradient gel as described above and stained with $\mathrm{KCl}$ as described by Hager and Burgess (10). The $60 \mathrm{kDa}$ polypeptide bands, reacting with

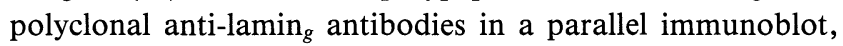
were excised out from several lanes. They were then placed in the large well of the sample cup of the ISCO sample concentrator (Model 1750). The sample cup and the inner compartment buffer composition was $0.057 \mathrm{M}$ Tris-acetate, $\mathrm{pH}$ 8.6/0.002 M EDTA $/ 0.001 \%$ SDS. The composition of the electrode buffer was $0.1 \mathrm{M}$ Tris-acetate, $\mathrm{pH} 8.6 / 0.002 \mathrm{M}$ EDTA/0.001\% SDS. Electroelution was carried out for $12 \mathrm{hr}$. at $4{ }^{\circ} \mathrm{C}$ following which all the fractions of the sample cup were collected and lyophilized. The residue was dissolved in double distilled water, dialyzed against $1 \%$ acetic acid and relyophilized.

For immunoblotting studies, proteins were transferred electrophoretically onto a nitrocellulose sheet according to the method of Towbin et al. (29) for $2 \mathrm{hr}$. at 1 Amp current using a Hoeffer Transphor electrophoresis unit. On the nitrocellulose sheet $\operatorname{lamin}_{g}$ antigen was detected by using HRP-conjugated goat anti-rabbit IgG (for polyclonal antibodies) or HRP-conjugated rabbit anti-mouse IgG (for monoclonal antibodies) as secondary antibodies at a dilution ranging from 1 : 500 to $1: 2000$.

Generation of monoclonal antibodies. For immunization of BALB/c mice, the lamin $g$ bands were cut out from a coomassie blue stained gel of sonication resistant nuclear associated proteins. A gel piece containing approximately $10 \mu \mathrm{g}$ of $\operatorname{lamin}_{g}$ was inserted subdermally on the dorsolateral side of 
the torso of each mouse through an incision which was sutured later. The mice were subjected to this protocol three times at 21 day intervals. Test serum was obtained from the orbital plexus 9th day after the third administration. Three days before the scheduled fusion, mice were injected intraperitoneally with approximately $20 \mu \mathrm{g}$ of the electroeluted antigen in saline. Splenocytes were fused with $\mathrm{Sp} 2 / \mathrm{O}$ mouse myeloma cells to generate hybridoma using standard procedures (32). The screening procedures used at various stages of generating monoclonal antibodies included ELISA (11), Dot-binding assay (12), solid phase radioimmuno assay (11) and finally the immunoblotting technique. One month after fusion, subcloning was carried out by the method of limiting dilution to obtain monoclonals.

Immunofluorescence microscopy. Indirect immunofluorescence studies on purified nuclear preparations were done according to the method of Mura and Stollar (20) with minor modifications. Freshly prepared nuclei were washed with TKM (50 mM Tris- $\mathrm{HCl}, \mathrm{pH} 7.5 / 25 \mathrm{mM} \mathrm{KCl} / 5 \mathrm{mM} \mathrm{MgCl}_{2}$ ) containing $0.2 \%$ Triton $\mathrm{X}-100$. The nuclear pellet was resuspended in TKM and filtered through 4 layers of cheese cloth. A small aliquot of the suspension was spread on a clean microscopic slide. After air drying, nuclei were fixed by adding a drop of fixative (freshly prepared $4 \%$ paraformaldehyde in $0.1 \mathrm{M}$ sucrose). The slides were kept aside for $5 \mathrm{~min}$, and were washed 3-4 times with TKM in a petri dish. Approximately, $200 \mu 1$ of the culture supernatant containing monoclonal antibodies was placed on the nuclear spread and incubated at room temperature for $1 \mathrm{hr}$. The slides were subsequently washed several times with TKM. A $1: 100$ dilution of FITC labelled goat anti-mouse IgG was then spread on the slide and incubated for $1 \mathrm{hr}$ at room temperature. The excess secondary antibody was washed with TKM. The nuclear spread was then air dried and mounted with 50\% glycerol in TKM and viewed under a Zeiss Axioplan microscope fitted with epifluorescence attachment. Photographs were taken with either ORWO NP27/ILFORD ASA 400 or KONICA ASA 400 colour films.

Immunohistochemical staining of seminiferous tubules. Testes from a 30 day old rat was excised and immediately immersed in Bouin's fixative. After 2 minutes, they were cut transversely into $3-4 \mathrm{~mm}$ pieces and immersed in the fixative for 8 hours. After washing and dehydration, paraffin blocks were made. Sections of 3-4 $\mu \mathrm{m}$ were then cut with the help of a microtome. After fixing these sections on a slide, they were further dewaxed in xylene for 3 minutes with two changes. The sections were then rehydrated by passing them through graded alcohol series including two changes in absolute ethanol ( 3 minutes each), 2 changes in $95 \%$ ethanol and 2 changes in $70 \%$ ethanol. After rinsing the sections in double distilled water, the sections were treated with $0.1 \%$ Triton X 100 in phosphate buffer saline (PBS) 2 times. Endogenous peroxidase activity was blocked by incubating the specimen with a solution containing 4 parts of methanol and 1 part of $3 \% \mathrm{H}_{2} \mathrm{O}_{2}$ for 20 minutes. The slide was then rinsed with PBS. Subsequently, sections were incubated with $1 \%$ normal goat serum in PBS for 10 minutes to ensure thorough blocking.

The sections were then separately incubated with either Mab $A_{11} D_{4}$ (neat supernatant) or normal mouse serum (1 : 1000 diluted in PBS) overnight at $4^{\circ} \mathrm{C}$.

After washing the sections 3 times with PBS (for 5 minutes each) they were incubated with rabbit anti-mouse peroxidase conjugate diluted $1: 50$ in PBS for 1 hour and were washed similar manner as mentioned earlier. The sections were then stained with Diaminobenzidine in PBS $(6 \mathrm{mg}$ DAB in $12 \mathrm{ml}$ of PBS). To this, $20 \mu \mathrm{l}$ of $30 \% \mathrm{H}_{2} \mathrm{O}_{2}$ was added. The sections were then washed in PBS and mounted in 50\% glycerol medium. Colour photographs of sections were taken using Kodak ASA 100 colour film using Zeiss Axioplan microscope. Alternatively, cryostat sections of rat testes were taken, fixed with ice cold acetone, incubated with Mab $A_{11} D_{4}$ and later with FITC-labelled rabbit anti-mouse IgG. Photographs were taken using a 400 ASA black and white film.

Double antibody staining of spermatogonial nuclei. $\mathrm{Nu}-$ clei from 10 day old rats were isolated in the same manner as mentioned earlier. A moderately thick suspension was smeared on a clean glass slide and fixed with $4 \%$ paraformaldehyde. The nuclei were incubated first with anti liver lamin B antibody and later with monoclonal anti $\operatorname{lamin}_{g}-A_{11} D_{4}$ with washes in between. Each incubation step was carried out for a period of $1 \mathrm{hr}$. Subsequently, after washing off excess antibody, the nuclei were incubated with rhodamine labelled goat anti rabbit IgG and later with FITC labelled goat anti mouse IgG. After thorough washing, the slide was air dried, mounted with 50\% glycerol in PBS and viewed under the fluorescence microscope. Colour photographs were taken of the phase contrast as well as the fluorescence pattern using different filters. The film used was Konica ASA 400 colour film.

Immunolocalization in surface spread pachytene spermatocytes. The seminiferous tubules from the testes of 40 day old rats were throughly minced in PBS and incubated on ice for 10 minutes. The supernatant was decanted and centrifuged at $3,500 \times \mathrm{g}$ for 10 minutes. After two washes with ice cold PBS, the cells were suspended in a small volume of PBS to give a medium thick suspension. A $100 \mu 1$ aliquot was placed on the top of a drop of $0.5 \%$ sodium chloride, on a piece of parafilm. After 1 minute, the spread nuclei were picked up on a clean microscopic slide and left to air dry for 5 minutes. Later, the specimen was fixed with $4 \%$ paraformaldehyde in $0.1 \mathrm{M}$ sucrose for 30 minutes. Subsequently, the slides were washed gently 3-4 times, with PBS. The nuclear spread was now

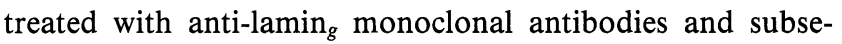
quently with FITC- labelled goat anti-mouse IgG as described above. After taking photographs of the phase contrast and the fluorescence pattern, the slide was processed for silver staining to visualize the synaptonemal complexes (9). A drop of $\mathrm{AgNO}_{3}$ solution $\left(1 \mathrm{~g} / \mathrm{ml}\right.$ in $\mathrm{H}_{2} \mathrm{O}$ ) was placed on the nuclear spread and incubated overnight at $60^{\circ} \mathrm{C}$ in a humidified chamber. The slide was then rinsed briefly in double distilled water and air dried. After mounting with 50\% glycerol, the same nucleus was relocated using pre-recorded coordinates and photo- 
graphed.

Analytical procedures. Protein estimations were done either by the method of Lowry et al. (18) or turbidometry (21).

\section{RESULTS}

Monoclonal antibodies against lamin $_{g}$ and their cross-reactivity with somatic lamins. A previous communication from our laboratory had shown that the $\operatorname{lamin}_{g}$, which is the sole component of the lamina structure of round spermatids, persists through spermiogenesis process and is even present in the epididymal sperm nucleus (28). $\operatorname{Lamin}_{g}$ was a component of the DNase 1 and high salt resistant fraction of sonication resistant nuclei (representing elongating and elongated spermatid nuclei). Although we used the same lamina isolation procedure to obtain this fraction, we have called it as the 'DNase 1 and high salt resistant fraction' since it is not clear as to what type of structure the lamina is transformed into, in the condensed nucleus of the sperm. The SDS-PAGE pattern of the polypeptides associated with this fraction isolated from sonication resistant nuclei is shown in Fig. 1A. We used the DNase 1 and high salt resistant fraction of sonication resistant nuclei as the source of the laming $_{g}$ antigen for raising monoclonal antibodies for two reasons (1). The sonication resistant nuclei can be isolated as a pure fraction from total testicular nuclei and (2) one can get enough starting material to run several gels wherefrom the polypeptide can be iso-
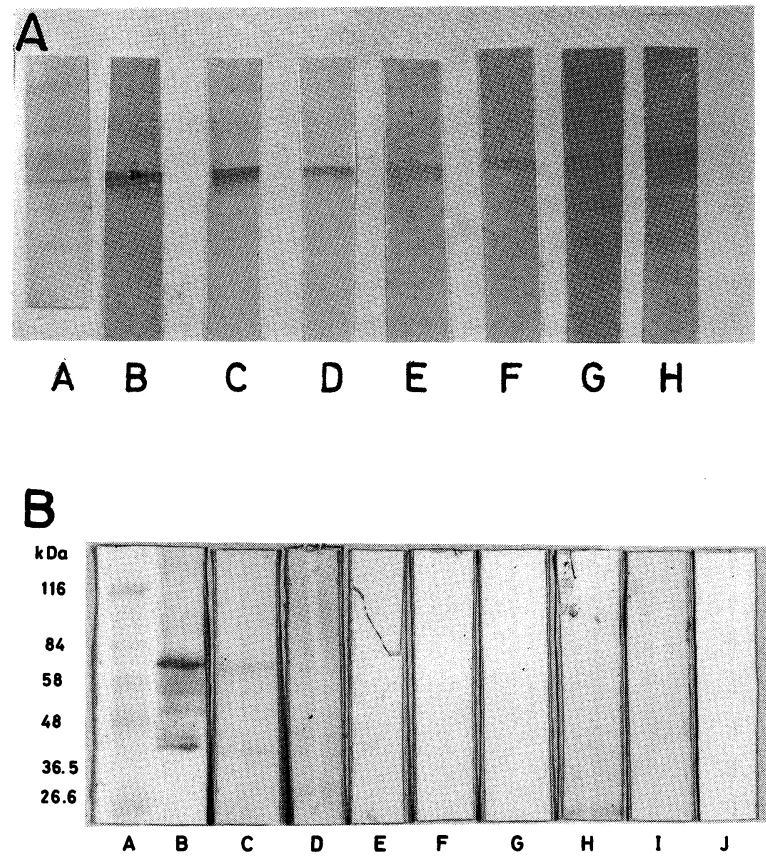

Fig. 2. Immunoblot analysis of $\operatorname{lamin}_{g}(\mathrm{~A})$ and liver lamina proteins

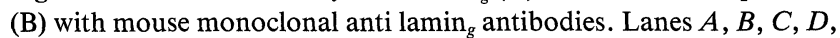
$E, F, G$ and $H$ in panel $A$ and lanes $C, D, E, F, G, H, I$ and $J$ in panel B represent immunoblots with Mabs $A_{11} C_{7}, A_{11} D_{4}, C_{1} F_{7}, C_{1} G_{8}, C_{8} B_{3}$, $C_{8} G_{5}, A_{2} C_{2}, D_{9} E_{6}$ respectively. Lane $A$ in panel $B$ is molecular weight markers and Lane $B$ is an immunoblot with rabbit polyclonal anti mouse lamin $B$ antibodies.
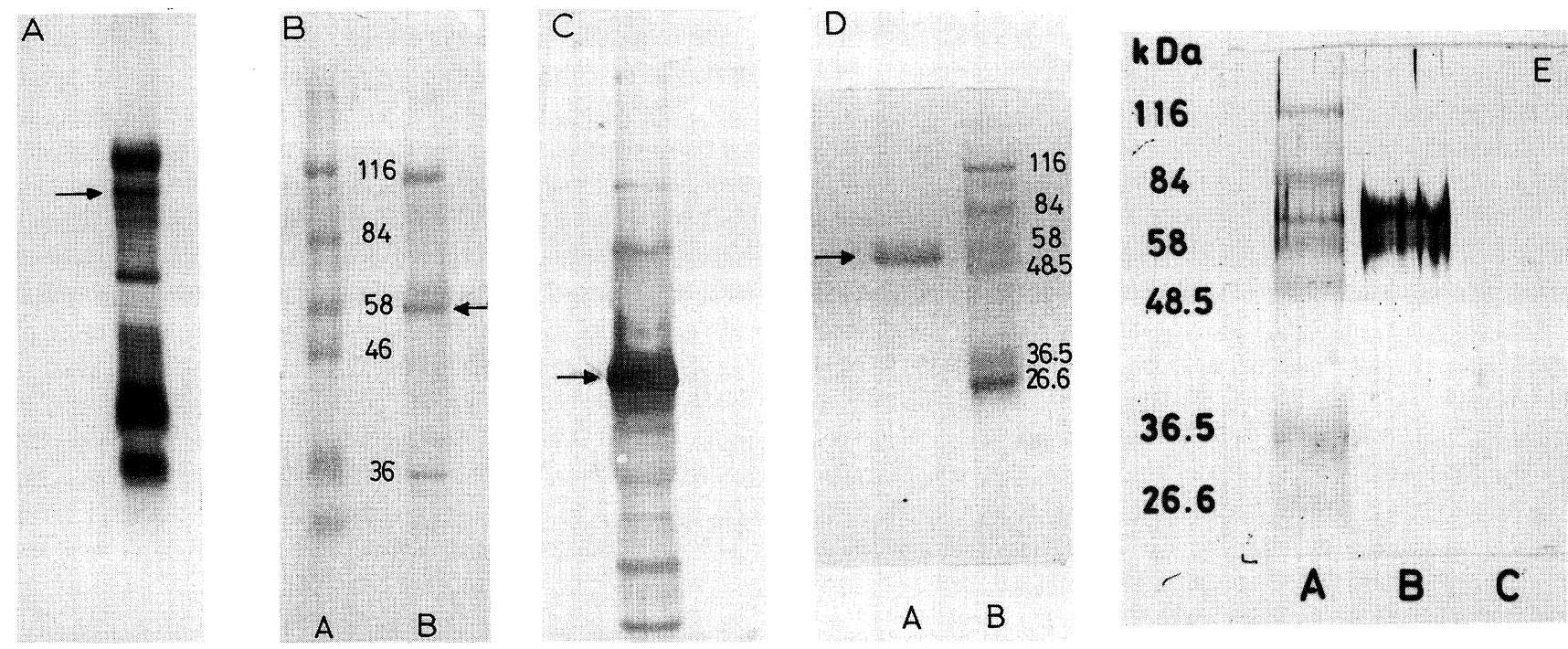

Fig. 1. Generation of mouse polyclonal antibodies against lamin . $_{\text {. }}$ (A) SDS-polyacrylamide gel electrophoretic pattern of sonication resistant nuclei of rat testes. The arrow represents the $60 \mathrm{kDa}$ lamin $_{g}$ band which was cut out and used for raising antibodies in mouse. (B) Immunoblot analysis of round spermatid lamina proteins with rabbit polyclonal antibodies (Sudhakar and Rao, 1991) Lane A, molecular weight markers;

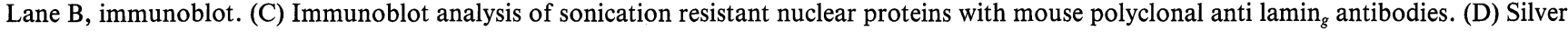

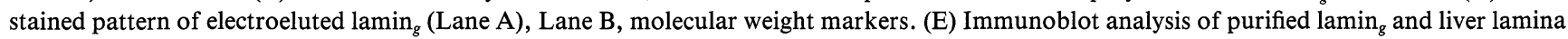
proteins with mouse polyclonal antibodies. Lane A, molecular weight markers; Lane B, electroeluted lamin $n_{g}$ C, liver lamina proteins. 
lated by electroelution after staining with $\mathrm{KCl}$.

We have tried several protocols for immunizing the mouse and found that subdermal placement of the gel band gave the best immune response. Fig. 1C shows the results of an immunoblot analysis of mouse polyclonal anti-lamin $_{g}$ antibodies in comparison with rabbit polyclonal anti-lamin ${ }_{g}$ antibodies, shown in Fig. 1B, which clearly indicates the efficiency of the immunization protocol we have used. The specificity of antigen recognition of the mouse polyclonal antibodies was then compared between (a) purified $\operatorname{lamin}_{g}$ (isolated by electroelution from $\mathrm{KCl}$ stained bands, the purity of which is shown in Fig. 1D) and (b) liver lamina proteins. The immunoblot pattern shown in Fig. 1E clearly shows that the antibodies are highly reactive with lamin ${ }_{g}$. Two very faint bands lighted up with the liver lamina proteins.
The upper band corresponds to lamin B while the bottom band may be either lamin $\mathrm{C}$ or a partially degraded lamin B itself. It may be worth mentioning here that even the rabbit anti-lamin ${ }_{g}$ polyclonal antibodies also showed a very faint cross reactivity with liver lamin B (27). In fact, we had inferred that lamin ${ }_{g}$ may be a variant form of lamin B based on the iodinated tryptic map analysis.

After confirming the presence of anti-lamin ${ }_{g}$ antibodies in the immunized mouse serum, we then proceeded to construct hybridoma cell lines and generate specific monoclonal antibodies. Eight monoclonal antibodies were obtained after various screening procedures and each of them was then finally checked for its reactivity with lamin $g$ by immunoblotting. The results of such an analysis is shown in Fig. 2A. It is clear that all the
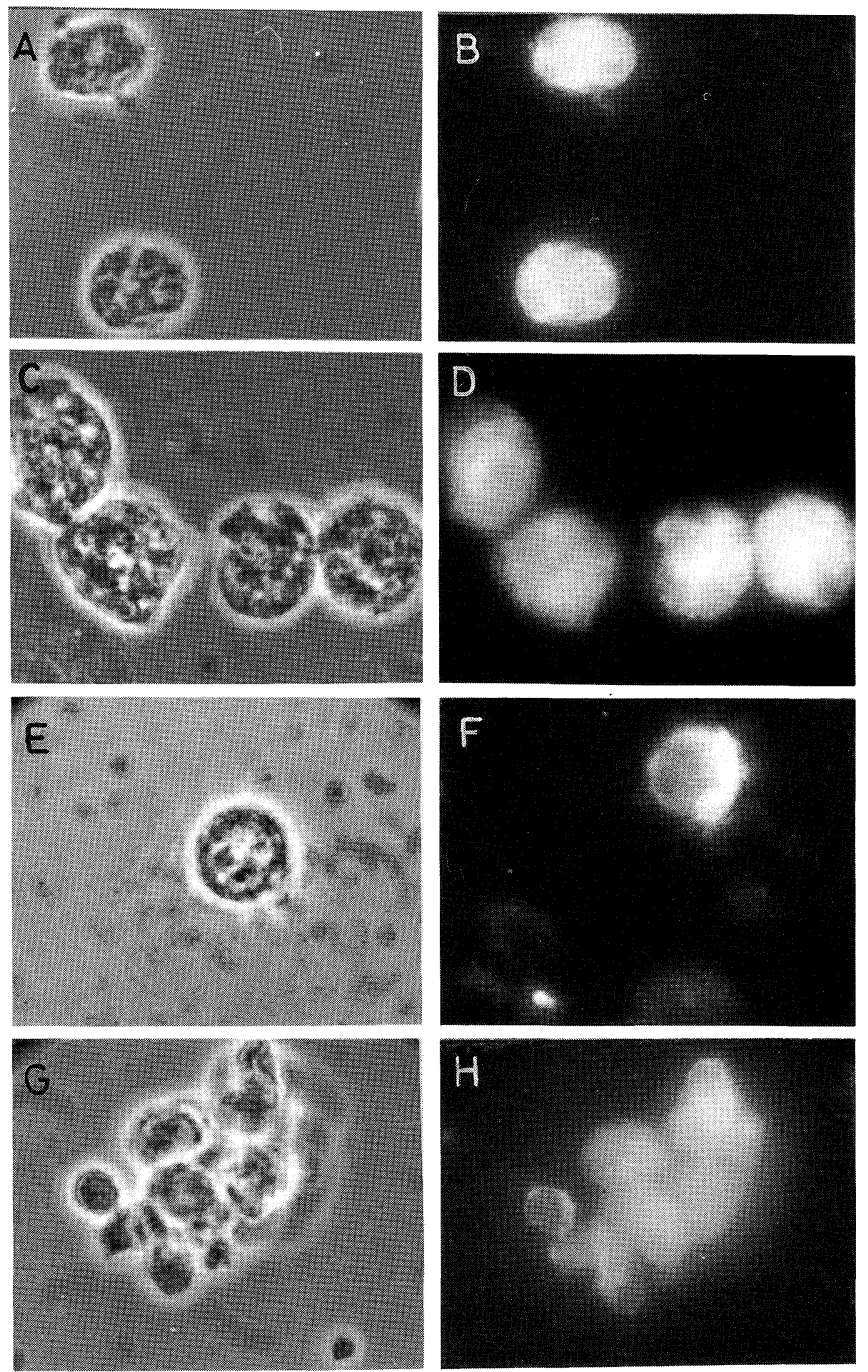
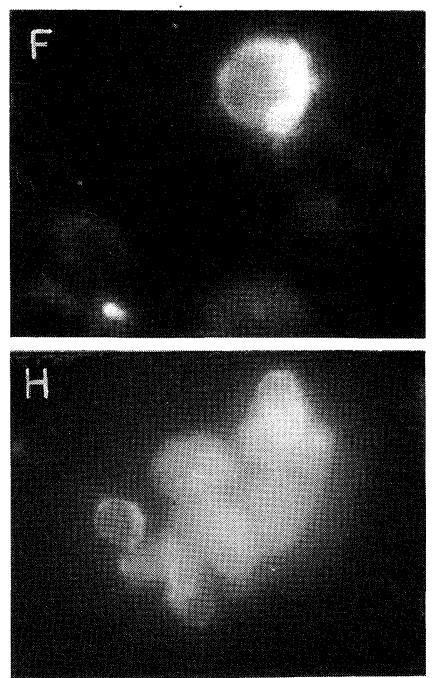
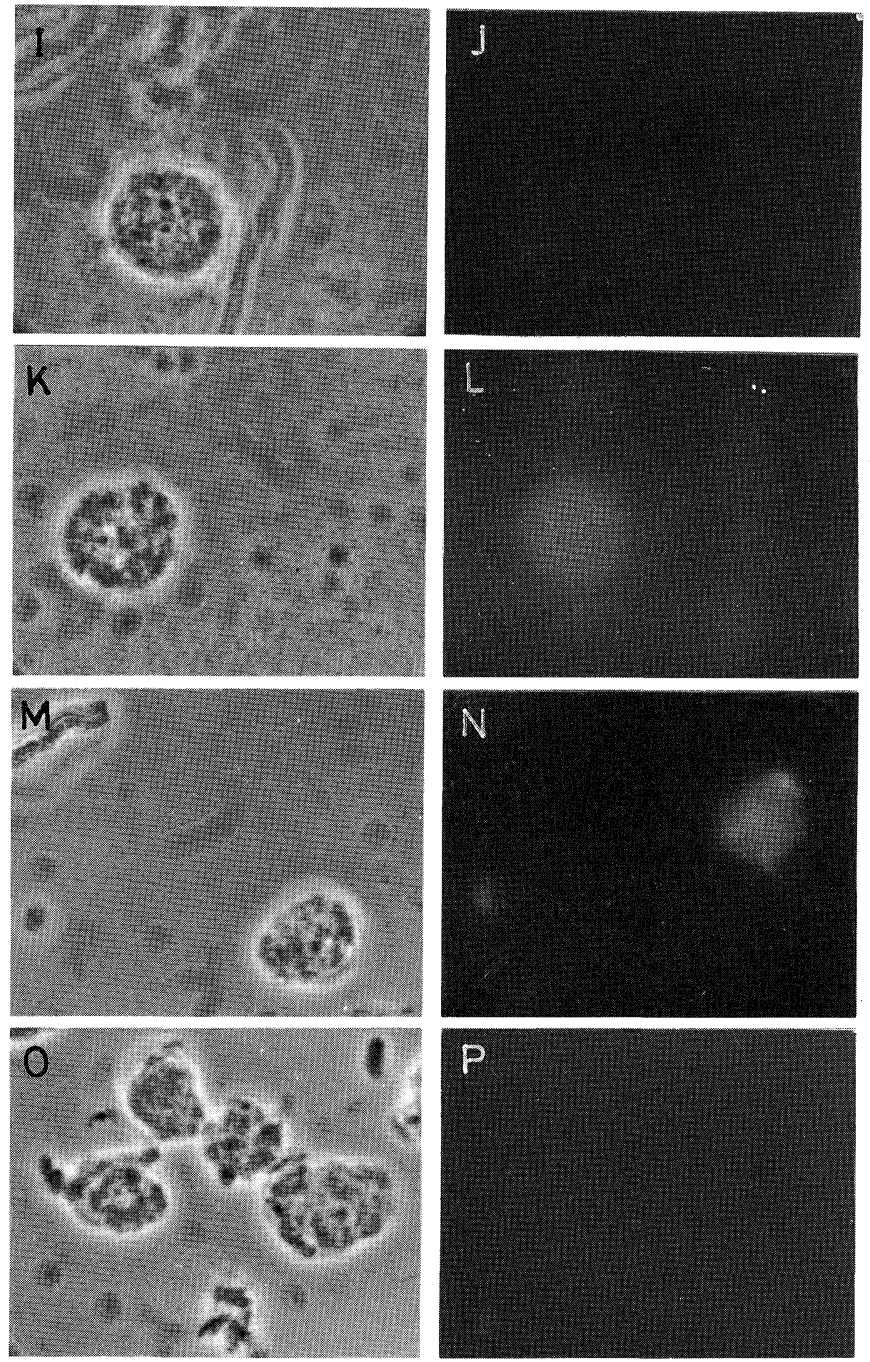

Fig. 3A. Indirect immunofluorescence analysis of pachytene spermatocyte nuclei with anti lamin $\operatorname{Mabs}_{g} A B\left(A_{11} C_{7}\right), C D\left(A_{11} D_{4}\right), E F\left(C_{1} F_{7}\right)$, $G H\left(C_{1} G_{8}\right), I J\left(C_{8} B_{3}\right), K L\left(C_{8} C_{5}\right), M N\left(A_{2} C_{2}\right)$ and $O P\left(D_{9} E_{6}\right)$. ACEGIKMO represent phase contrast micrographs while BDFHJLNP represent the fluorescence pattern, $\times 1,000$. 
eight monoclonal antibodies reacted specifically with the lamin $_{g}$ polypeptide, among with $A_{11} D_{4}, C_{1} F_{7}$ and $C_{1} G_{8}$ showed strong reactions. The differences in the intensity of reaction observed in the immunoblot analysis may reflect differences in the affinities of the monoclonal antibodies with the antigen. Occasionally, there was also a reaction with a band just below the $60 \mathrm{kDa} \operatorname{lamin}_{g}$ band. Since this was not reproducible in all the immunoblots, it may probably represent partially degraded lam$\mathrm{in}_{\mathrm{g}}$. All the monoclonal antibodies were then checked for their cross-reactivity with the liver lamina proteins. The results of these immunoblotting studies are shown in Fig. 2B. As a positive control, we have used rabbit anti-mouse lamin B polyclonal antibodies (courtesy of Dr. Veena Parnaik, CCMB, Hyderabad) which is shown in lane B. Among the monoclonals, only $A_{11} D_{4}$ and $A_{11} C_{7}$ showed a very faint reaction with somatic lamin $\mathrm{B}$. It has been observed that the strongest epitope in lamin B
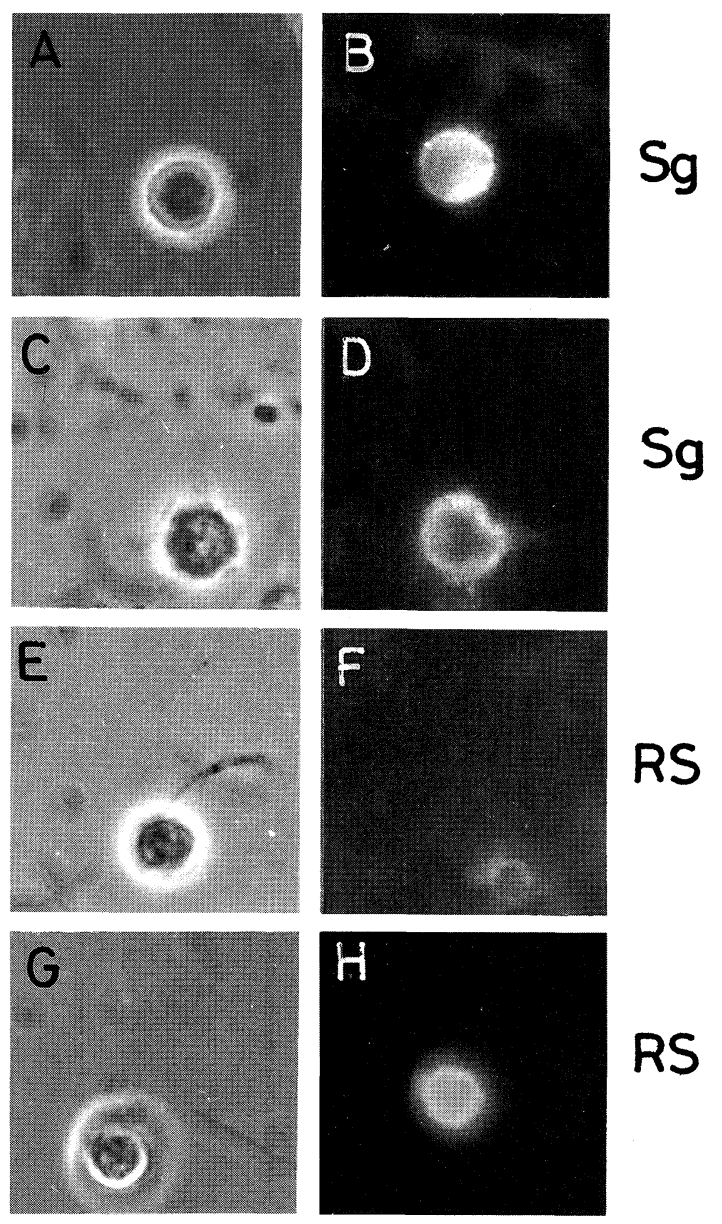

Fig. 3B. Indirect immunofluorescence analysis of spermatogonia and round spermatid nuclei with $A_{11} C_{7}\left(\mathrm{~A}, \mathrm{~B}, \mathrm{E}\right.$ and F) and $A_{11} D_{4}(\mathrm{C}$, $\mathrm{D}, \mathrm{G}$ and $\mathrm{H}$ ) anti lamin $g$ Mabs. A, C, E and G represent phase contrast micrographs while $\mathrm{B}, \mathrm{D}, \mathrm{F}$ and $\mathrm{H}$ represent the fluorescence pattern, $\times 1,000$. lies in a $46 \mathrm{kDa}$ chymotryptic fragment of lamin $\mathrm{B}(3)$. Since the two monoclonals $A_{11} D_{4}$ and $A_{11} C_{7}$ showed only a faint reaction with lamin $\mathrm{B}$, it is possible that this epitope is extensively modified in $\operatorname{lamin}_{g}$. The observation that the other six monoclonals did not react with lamin B at all, indicates probably that they might recognize unique epitope(s) in $\operatorname{lamin}_{g}$ which are absent in lamin B.

Immunofluorescence studies on the localization pattern of laming in germ cells using Mabs. Earlier, we had made a detailed study on the localization of lamin ${ }_{g}$ in spermatogonia, spermatocytes and round spermatids by the indirect immunofluorescence technique using rabbit polyclonal antibodies. Now, since we have obtained 8 different monoclonals recognizing the $\operatorname{lamin}_{g}$ antigen, we have repeated the indirect immunofluorescence studies with each of the monoclonals to get more insight on the pattern of localization of this antigen in the spermatogenic cells. Fig. 3A shows the fluorescence pattern obtained with pachytene spermatocyte nuclei. Among the $8 \mathrm{Mabs}, A_{11} C_{7}, A_{11} D_{4}$ and $C_{1} G_{8}$ showed the strongest fluorescence pattern. The fluorescence pattern is very similar to the ones we have reported earlier using polyclonal antibodies in that it is patchy in appearance with a discontinuous fluorescence pattern around the nuclear periphery. Furthermore, the strongest fluorescence within the nucleus was localized to the phase dense regions. The fluorescence pattern observed with Mabs $C_{1} F_{7}$ and $A_{2} C_{2}$ was much less intense as compared to the ones observed with $A_{11} C_{7}, A_{11} D_{4}$ and $C_{1} G_{8}$. On the other hand, there was negligible fluorescence observed with Mabs $C_{8} B_{3}, C_{8} C_{5}$ and $D_{9} E_{6}$. It is interesting to note here that although all the 8 Mabs showed reaction with the $\operatorname{lamin}_{g}$ antigen in the immunoblot analysis

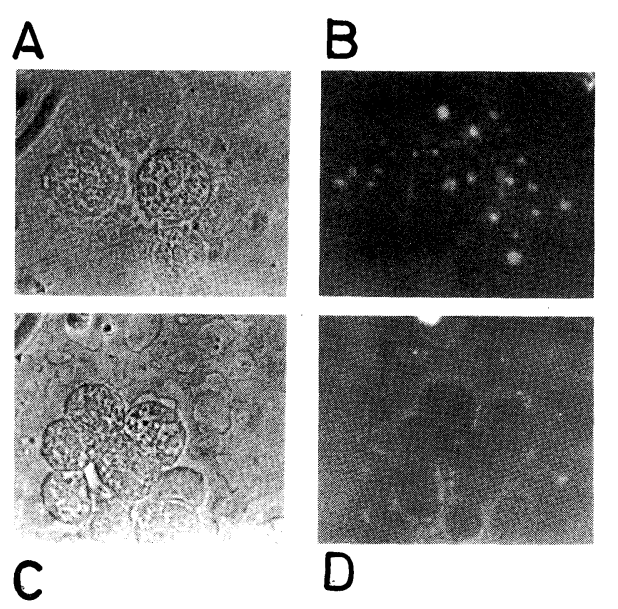

Fig. 4. Indirect immunofluorescence pattern of liver nuclei with anti lamin Mabs, $_{A_{11}} C_{7}(A, B)$ and $A_{11} D_{4}(C, D)$. A and $\mathrm{C}$ represent phase contrast micrographs while $\mathrm{B}$ and $\mathrm{D}$ represent the fluorescence pattern, $\times 1,000$. 
(Fig. 2A), only 5 Mabs showed significant fluorescence in the pachytene nucleus. These results, therefore, suggest that the two sets of Mabs may be recognizing two different regions in the protein. The epitope(s) recognized by Mabs $C_{8} B_{3}, C_{8} C_{5}$ and $D_{9} E_{6}$ may not be accessible in $\operatorname{lamin}_{g}$ in the lamina structure while it is accessible in the immunoblot analysis.

Among the 8 Mabs, $A_{11} D_{4}$ and $A_{11} C_{7}$ which showed strongest fluorescence in pachytene spermatocyte nucleus were then used to screen the pre-meiotic spermatogonial nuclei and post-meiotic round spermatid nuclei. As can be seen from the immunofluorescence pattern shown in Fig. 3B, it is clear that both the Mabs decorated the nuclear periphery characteristic of the laminar fluorescence. The other Mabs which were negative with respect to fluorescence in the pachytene nucleus $\left(D_{9} E_{6}\right.$, $C_{8} B_{3}, C_{8} C_{5}$ ) were also negative in spermatogonial and round spermatid nuclei (data not shown). While both the Mabs exhibited comparable reactivities with the spermatogonial nuclei the immunofluorescence intensity was much stronger in round spermatid nuclei with the Mab $A_{11} D_{4}$ as compared to the Mab $A_{11} C_{7}$. A closer look at the fluorescence pattern of pachytene nuclei with these two Mabs also reveal that the intensity with $A_{11} D_{4}$ is a little stronger than $A_{11} C_{7}$. It may be pertinent to mention here that the Mab $A_{11} D_{4}$ also exhibited a stronger reaction than Mab $A_{11} C_{7}$ with the laming even in the immunoblot. These observations suggest that the Mabs $A_{11} C_{7}$ and $A_{11} D_{4}$ may be recognising different epitopes in $\operatorname{lamin}_{g}$.

Since Mabs $A_{11} D_{4}$ and $A_{11} C_{7}$ showed a faint cross-reactivity with somatic lamin $\mathrm{B}$ in the immunoblot analysis (Fig. 2B), we were curious to see whether they can immunostain the liver nuclei. The results shown in Fig. 4 indicate that both the Mabs yielded only faint laminar fluorescence, in contrast to the strong laminar fluorescence observed in the positive control using anti-lamin B antibodies (Fig. 5).

All the immunofluorescent studies described above and in our earlier reports $(27,28)$ were carried out on isolated testicular nuclei. In order to confirm that these

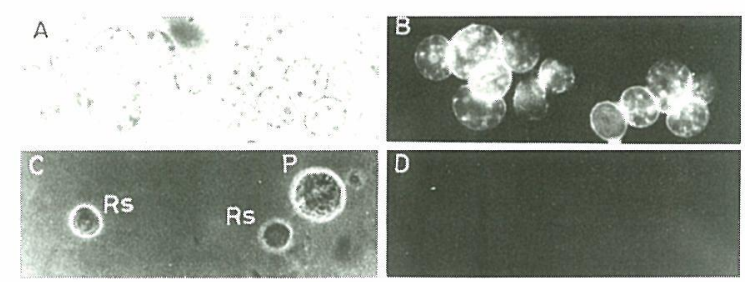

Fig. 5. Indirect immunofluorescence pattern of liver nuclei (A and $B$ ) and germ cells ( $C$ and $D)$ with rabbit polyclonal anti lamin B antibodies. A and $\mathrm{C}$ represent phase contrast micrographs while $\mathrm{B}$ and $\mathrm{D}$ represent the fluorescence pattern. $\mathrm{P}$, pachytene; Rs, round spermatids. $\times 1,000$.
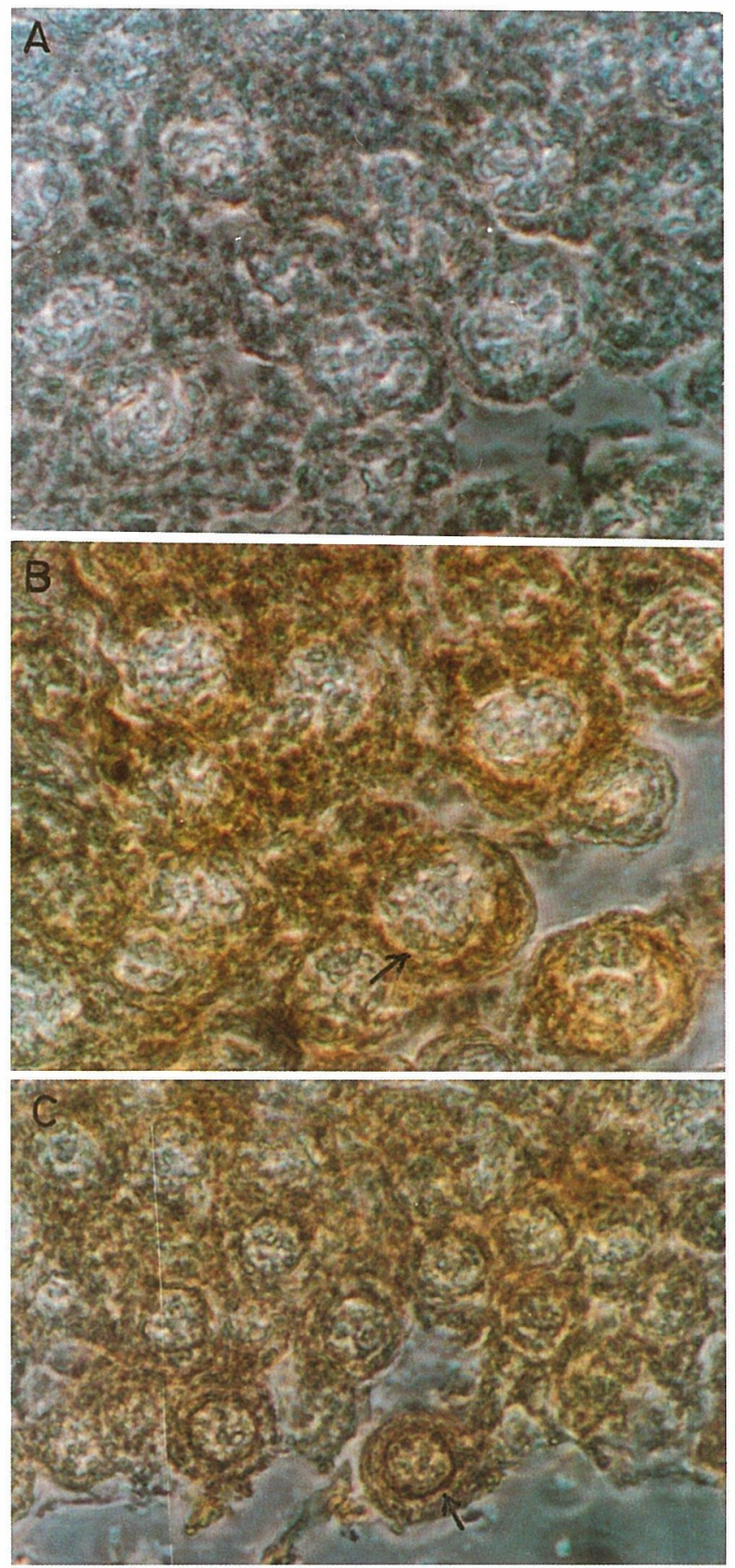

Fig. 6. Immunohistochemical localization of $\operatorname{lamin}_{g}$ in thin sections of seminiferous tubules. A section treated with normal mouse serum ( $1: 1000$ dilution in PBS). B and C sections treated with Mab $A_{11} D_{4}$. Subsequently, the sections were processed for peroxidase staining as described in Methods. D represents a thin section stained with FITC secondary antibodies. A, B and C; $\times 1,000$ magnification and D, $\times 400$ magnification. Shaded arrows point to round spermatids. Blank arrows point to pachytene nuclei. 
Table I. CharaCterisation OF MONOCLONAL ANTIBODIES AGAINST the GERM CELL SPECIFIC LAMin.

\begin{tabular}{|c|c|c|c|c|c|c|c|}
\hline \multirow{2}{*}{$\begin{array}{l}\text { Monoclonal } \\
\text { Antibody }\end{array}$} & \multirow{2}{*}{ Isotype } & \multirow{2}{*}{$\begin{array}{l}\text { Reactivity } \\
\text { with } \mathrm{L}_{g} \text { in } \\
\text { immunoblot }\end{array}$} & \multirow{2}{*}{$\begin{array}{c}\text { Reactivity } \\
\text { with LLB in } \\
\text { immunoblot }\end{array}$} & \multicolumn{4}{|c|}{ Fluorescence in Nuclei } \\
\hline & & & & liver & $\mathrm{Sg}$ & Pac & Rs \\
\hline$A_{11} C_{7}$ & $\operatorname{Ig} G_{3}$ & * & $*$ & Faint dotted & $\begin{array}{c}* \\
\text { Laminar }\end{array}$ & $\begin{array}{c}\text { Patchy } \\
* *\end{array}$ & $\begin{array}{c}* \\
\text { Laminar }\end{array}$ \\
\hline$A_{11} D_{4}$ & $\operatorname{Ig} G_{2 \mathrm{~b}}$ & $* *$ & Faint & Faint laminar & $\begin{array}{c}* \\
\text { Laminar }\end{array}$ & $\begin{array}{c}\text { Patchy } \\
* * *\end{array}$ & $\begin{array}{c}* * \\
\text { Laminar }\end{array}$ \\
\hline$C_{1} F_{7}$ & $\operatorname{Ig} G_{2 \mathrm{~b}}$ & $* *$ & - & - & - & $\begin{array}{c}\text { Patchy } \\
*\end{array}$ & - \\
\hline$C_{1} G_{8}$ & - & $* *$ & - & - & - & $\begin{array}{c}\text { Patchy } \\
* *\end{array}$ & - \\
\hline$C_{8} B_{3}$ & $\operatorname{Ig} G_{1}$ & * & - & - & - & - & - \\
\hline$C_{8} C_{5}$ & $\operatorname{Ig} G_{1}$ & * & - & - & - & - & - \\
\hline$A_{2} C_{2}$ & $\operatorname{Ig} G_{2 \mathrm{~b}}$ & $*$ & - & - & - & Patchy & - \\
\hline$D_{9} E_{6}$ & $\operatorname{Ig} G_{1}$ & $*$ & - & - & - & - & - \\
\hline
\end{tabular}

results are not an artefact, we have also carried out localization of the $\operatorname{lamin}_{g}$ antigen in thin sections using Mab $A_{11} D_{4}$. These results are presented in Fig. 6. It is clear that the antigen shows a laminar localization in both pachytene spermatocyte (Figs. 6B and 6D) and round spermatid (Fig. 6C) nuclei, confirming the observations made with isolated nuclei.

As mentioned in the Introduction, Stick and Schwarz (26) could not detect lamina proteins either in pachytene spermatocytes or in round spermatids. We had argued in our previous paper that the lack of detection of the lamina structure in their studies was due to the use of antibodies against somatic lamin B. In order to make sure that there are no experimental artefacts in our studies using both the polyclonal antibodies (27) and monoclonal antibodies (present study), we have carried out indirect immunofluorescence studies of both liver and germ cell nuclei with polyclonal anti-mouse lamin B antibodies. These results presented in Fig. 5 clearly show that while these antibodies exhibited strong laminar fluorescence in liver nuclei, the same antibodies did not decorate nuclei either from pachytene spermatocytes or round spermatids. The punctate kind of fluorescence of the lamina structure has also been observed by Burke et al. (3). Thus, these results confirm our earlier conclusion that a germ cell specific lamin which exhibits
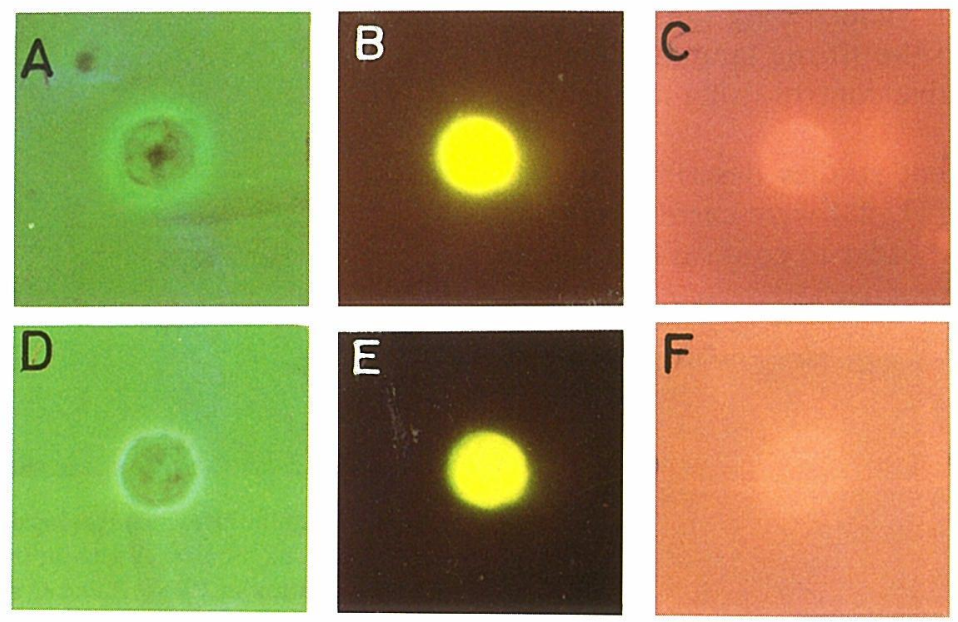

Fig. 7. Colocalization of lamin $B$ and lamin $_{g}$ in spermatogonia. Spermatogonial nuclei were doubly stained with rabbit polyclonal anti lamin $\mathrm{B}$ antibodies and anti lamin $\operatorname{Mab}_{11} D_{4}$. The lamin ${ }_{g}$ and lamin B antigens were detected by FITC labelled anti mouse IgG in goat and rhodamine labelled anti rabbit IgG in goat respectively. A, B, C and D, E, F represent two different spermatogonial nuclei. A and D represent the phase contrast micrographs. B and $\mathrm{E}$ represent the fluorescence pattern of FITC conjugated secondary antibody while $\mathrm{C}$ and $\mathrm{F}$ represent the fluorescence pattern of rhodamine conjugated secondary antibody. 

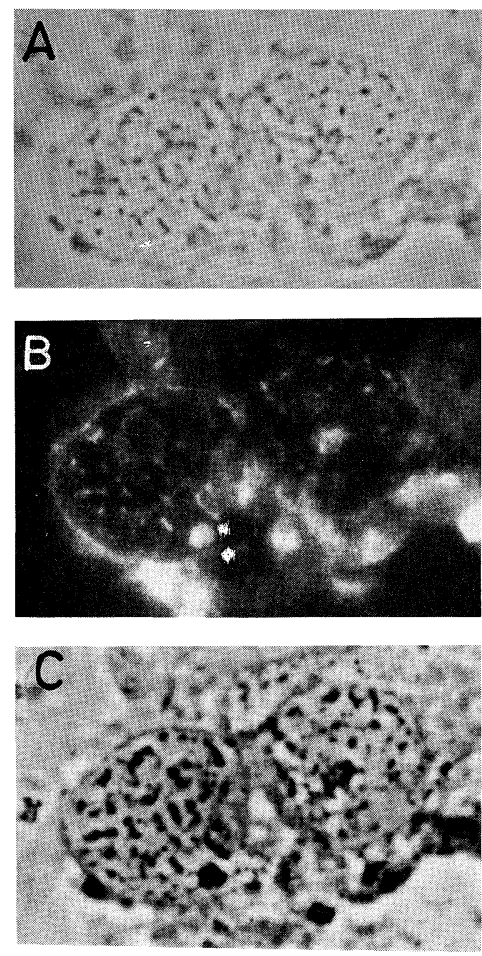

Fig. 8. Localization of $\operatorname{lamin}_{g}$ antigen in surface spread spermatocytes. Surface spread spermatocytes were prepared as described in Methods. The microscope slide was first treated with Mab $A_{11} D_{4}$ and FITC-conjugated secondary antibody and visualized for the fluorescence pattern (B). Subsequently the spread was processed for $\mathrm{AgNO}_{3}$ staining to visualize the S.Cs (C) A, phase contrast micrograph. The arrow in the phase contrast micrograph indicates two phase dense regions corresponding to the two telomeric ends of the chromosomes as is evident from the $\mathrm{Ag}$-stained pattern in $(C)$. The arrow in $\mathrm{C}$ points to the dense body which is also immunostained with Mab $A_{11} D_{4}$.

very little cross-reactivity with somatic lamin B is present in meiotic and post-meiotic germ cells.

A summary of the observations made with all the 8 Mabs with respect to the fluorescence pattern and the immunoblot analysis of $\operatorname{lamin}_{g}$ antigen is presented in Table I. The main conclusions from these studies agree very well with those drawn from studies done with polyclonal antibodies made in our previous study. Namely, (a) $\operatorname{lamin}_{g}$ antigen is a part of the lamina structure in the pre-meiotic germ cells, (b) As the germ cells enter meiotic prophase, the lamin ${ }_{g}$ antigen is retained in the pachytene nucleus even though the lamina structure has disassembled and (c) following meiotic division, the lamina structure is reformed using lamin ${ }_{g}$ as the only lamina component.

Co-localization of lamin B and lamin $_{g}$ in the spermatogonial cells. In the model proposed by us in our previous paper (27), we had speculated that both the somatic lamins and $\operatorname{lamin}_{g}$ coexist in the nuclei of pre-mei- otic spermatogonial cells. Upon entry into the meiotic prophase, only lamin ${ }_{g}$ is retained and the same is relocalized into the lamina structure in round spermatids. However, we had not provided evidence for the coexistence of the lamin B and lamin ${ }_{g}$ within the same nucleus. Now since we have shown in this paper that anti-lamin B antibodies do not react with $\operatorname{lamin}_{g}$ (Fig. 5) and anti$\operatorname{lamin}_{g}$ antibodies have negligible cross- reactivity with lamin B (Fig. 2B and Fig. 4), we have carried out double antibody labelling of spermatogonial nuclei. For this purpose, nuclei were isolated from the tests of 12 day old rats (containing predominantly spermatogonia and a few preleptotene spermatocytes) and then subjected to double antibody labelling as mentioned in Methods. The lamin B was followed by Rhodamine labelled anti

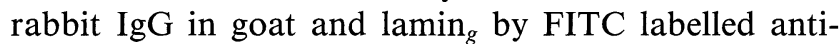
mouse IgG in goat as secondary antibodies. The immunofluorescence pattern shown in Fig. 7 shows that both lamin B and $\operatorname{lamin}_{g}$ coexist within the same spermatogonial nucleus. A more confirmatory evidence for this colocalization needs to be obtained by immunoelectronmicroscopic studies.

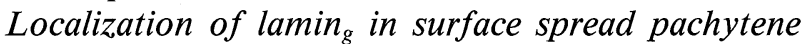
spermatocytes. An event concomittant to the disassembly process of the nuclear lamina structure during meiotic prophase is the appearance of synaptonemal complexes (SC). The lamin $_{g}$ antigen was coisolated with synaptonemal complexes, from rat pachytene spermatocytes (27). However, the fluorescence patternin the pachytene nucleus did not indicate that it is localized to either lateral elements or central elements of SCs. Both the immunofluorescence and preliminary immuno-electron microscopic studies showed it to be localized at the phase dense regions within the nucleus. These regions were speculated to correspond to the attachment plaque regions of the telomeric ends of the chromosomes through which they are attached to the nuclear membrane. To gain a further insight into the localization of $\operatorname{lamin}_{g}$ antigen, we have carried out immunolocalization studies in surface spread pachytene spermatocytes. For this purpose, testicular germ cells were given a mild hypotonic treatment $(0.5 \% \mathrm{NaCl})$ and then fixed with $4 \%$ paraformaldehyde. The spread was first reacted with $A_{11} D_{4}$ Mab and subsequently with FITC labelled goat anti-mouse IgG. The fluorescence pattern was recorded and then the same slide was processed for silver staining to visualize the SCs. A similar technique has been used by Dresser and Moses (4). Fig. 8 shows the results of such an experiment. Again, the fluorescence is observed at phase dense regions (Fig. 8B) in addition to a discontinuous fluorescence along the periphery of the nucleus. From a comparison of the phase contrast microgragh (Fig. 8A) with that of the silver stained micrograph (Fig. 8C), it is very clear that the phase dense regions correspond to the telomeric ends of the paired homolo- 
gous chromosomes. The fluorescence is also observed at the "dense body" region. The significance of these results is discussed below.

\section{DISCUSSION}

The present investigation is a continuation of our studies on the immunological characterization of the germ cell specific lamin . In our previous study using rabbit polyclonal antibodies, we had made several interesting observations (28). They were (a) $\operatorname{lamin}_{g}$ is retained in the pachytene spermatocytes even though the lamina structure is believed to be absent in these meiotic prophase cells (26), (b) the lamina structure of haploid gametes is made up solely of this $\operatorname{lamin}_{g}$ and (c) lamin is related but not identical to somatic lamin B. To gain further insight into the nature of $\operatorname{lamin}_{g}$ protein, we have produced and characterized 8 monoclonal antibodies. Although all these monoclonal antibodies reacted strongly with $\operatorname{lamin}_{g}$ in an immunoblot analysis, based on their reactivity with the antigen in immunofluorescence studies, they can be classified into 3 groups: (a) those showing a strong fluorescence $\left(A_{11} D_{4}, A_{11} C_{7}\right.$, $C_{1} G_{8}$ ); (b) those showing a medium intensity of fluorescence $\left(C_{1} F_{7}, A_{2} C_{2}\right)$; and (c) those showing no fluorescence at all in the germ cells $\left(C_{8} B_{3}, C_{8} C_{5}, D_{9} E_{6}\right)$. It is interesting to note that the monoclonal antibodies showing a strong fluorescence with germ cells, showed a very faint reaction with the laminar structure of liver nuclei (Fig. 4) and also with lamin B in an immunoblot analysis (Fig. 2B). These observations, in addition to strengthening our above conclusion (c), also suggest that there is only a small degree of homology in the epitope being recognized by these monoclonal antibodies in lamin ${ }_{g}$ and lamin B. The epitopes recognised by the other two sets of monoclonal antibodies ( $b$ and c) may be unique to $\operatorname{lamin}_{g}$ which are absent in lamin B. The different levels of fluorescence generated by the three sets of monoclonal antibodies may reflect the differential accessibility of the respectivey epitopes in germ cells.

The lack of cross-reactivity, or, in some cases a low level of cross-reactivity of the anti-lamin monoclonal antibodies with lamin $\mathrm{B}$ is rather intriguing, particularly considering the fact that somatic lamins A, B and C are immunologically related as reported by Raymond and Gagnon (23). These workers have obtained 2 monoclonal antibodies (34B6 and 55B3) which reacted with all the three lamins A, B and C while another (36C2) reacted only with lamins $A$ and $C$. One of the 2 monoclonal antibodies 31B5 reacted specifically with lamin B only. Using an IgM monoclonal antibody, Burke et al. (3) had earlier shown that it reacts with all the three somatic lamins and this epitope is localized to a $46 \mathrm{kDa}$ chymotryptic fragment generated from each of the lam- ins. In addition to their immunological relatedness, the lamins also share antigenic determinants with intermediate filament proteins (23). It may be pertinent to point out here, that even the rabbit anti-lamin $g$ polyclonal antibodies reacted very faintly with lamin B (27). Conversely, the experiments described here with anti somatic lamin B antibodies revealed that they do not light up germ cells in immunofluorescence studies (Fig. 5).

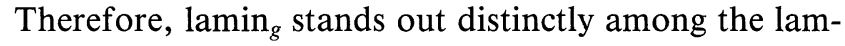
ina proteins and it becomes very crucial to identify and characterize the epitopes recognized by the anti-lamin ${ }_{g}$ monoclonal antibodies that we have generated. These studies are now in progress in our laboratory.

An important observation made in this study is the colocalization of lamin B and $\operatorname{lamin}_{g}$ in spermatogonial cells, which supports the model proposed by us (27). Although both the somatic lamin B and $\operatorname{lamin}_{g}$ are present in the premeiotic germ cells, only lamin $_{g}$ is selectively retained in the pachytene nucleus. Therefore, we believe that $\operatorname{lamin}_{g}$ must have some unique properties that are absent in lamin B in order to facilitate the organization of the meiotic chromosomes. Interestingly, lamin B is constitutively expressed in all the tissues while lamins $\mathrm{A} / \mathrm{C}$ are acquired only upon tissue maturation (25). Recently, we have shown that lamin ${ }_{g}$ is evolutionarily conserved in the germ cells of many eukaryotic cells (28). This antigen, however was not observed in somatic cells of these organisms, stressing the functional importance of lamin $_{g}$ in the germ cells. It is very essential, therefore, to compare the sequence organization and molecu-

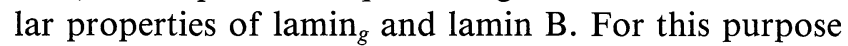
we are presently characterizing the cDNA clone for lam$\mathrm{in}_{g}$ which should provide valuable information to answer many of the questions raised above.

Another series of experiments described in the present study are concerned with the localization of lamin $g$ in pachytene spermatocytes which included both isolated nuclei as well as thin sections of seminiferous tubules period. The results obtained with monoclonal antibodies confirm our earlier conclusions, namely it is localized in the phase dense regions of the pachytene spermatocyte nucleus. Our results on the double staining of surface spread pachytene spermatocytes with FITC and silver have clearly shown that the phase dense regions correspond to the telomeric ends of the meiotic chromosomes. A question that arises at this juncture is why lam$\mathrm{in}_{g}$ is retained in the pachytene nucleus when there is no lamina structure. However, there is one important difference in the organization of nuclear structure between a mitotic cell and a meiotic cell particularly in mammals. Although the lamina structure is disassembled in both the cases, the nuclear membrane is retained in the meiotic cell nucleus but not in the mitotic cell. Both ends of the synaptonemal complex appear to be in contact with the inner nuclear membrane in many organ- 
isms (31). Therefore, we believe that the retention of $\operatorname{lamin}_{g}$ is necessary to facilitate the attachment of the telomeric ends of the chromosomes to the inner nuclear membrane. In fact, lamin B is believed to provide the anchorage sites for the surrounding lipid bilayer membrane (7). The regions of attachment of the telomeric ends of the chromosomes to the nuclear membrane have been termed cytologically as "attachment plaques" or "chromosomal bouquets". Recently, Klein et al. (14) have shown that RAP1 (repressor activator protein 1) is localized similarly to the telomeric ends of meiotic chromosomes in Saccharomyces cerevesiae using similar technique. The functional significance of the localization of RAP1 at telomeric ends, however, is not known. In addition to the staining of the telomeric ends of the chromosomes, we have also observed a faint discontinuous fluorescence pattern around the nuclear periphery (Fig. 8) which was also evident in the immunohistochemical staining of thin sections of seminiferous tubules (Fig. 6B and 6D). Therefore we believe that there are remnants of a lamina structure in the meiotic prophase nucleus attached to the inner nuclear membrane. Recently Vester et al. (1993) have also observed using a broad specificity antibody, lamina structure in rat spermatocytes involving a lamin protein that is closely related to lamin B which is in conformity with our findings.

Recently we have also come across a paper by Furukawa and Hotta (6) describing the isolation and characterization of a cDNA clone for a germ cell specific lamin in mouse. They have termed it as lamin B3 which arises out of alternate splicing from lamin B2. It remains to be seen whether the $\operatorname{lamin}_{g}$ that we have described in the rat is the same protein as lamin B3 of mouse.

Acknowledgement. This work was financially supported by grants from the Department of Biotechnology, New Delhi. The generous gift of rabbit polyclonal anti mouse lamin B antibodies from Dr. Veena Parnaik, CCMB, Hyderabad, is gratefully acknowledged.

\section{REFERENCES}

1. Behal, A., KulKarni, P., and Rao, M.R.S. 1987. Identification of a meiotic prophase-specific nuclear matrix protein in the rat. J. Biol. Chem., 262: 10898-10902.

2. Bhown, A.S., Mole, J.E., Hunter, F., and Bennett, J.C. 1980. High sensitivity sequence determination of proteins quantitatively recovered from sodium dodecyl sulphate gels using an improved electrodialysis procedure. Anal. Biochem., 103: $184-190$.

3. Burke, B., Tooze, J., and Warren, G. 1983. A monoclonal antibody which recognises each of the nuclear lamin polypeptides in mammalian cells. EMBO J., 2: 361-367.

4. Dresser, M.E. and Moses, M.J. 1980. Synaptonemal complex karyotyping in spermatocytes of the chinese hamster (Cricetulus griseus). IV. Light and electron microscopy of synapsis and nucleolar development by silver staining. Chromosoma, (Berl) 76: 1-22.

5. Dwyer, N. and Blobel, G. 1976. A modified procedure for the isolation of a pore complex-lamina fraction from rat liver nuclei. J. Cell Biol., 70: 581-591.

6. Furukawa, K. and Hotta, Y. 1993. cDNA cloning of a germ cell specific lamin $B_{3}$ from mouse spermatocytes and analysis of its function by ectopic expression in somatic cells. EMBO J., 12: 97-106.

7. Gerace, L., Blum, A., and Blobel, G. 1978. Immunocytochemical localization of the major polypeptides of the nuclear pore complex lamina fraction. Interphase and mitotic distribution. J. Cell Biol., 79: 546-566.

8. Gerace, L. and Blobel, G. 1980. The nuclear envelope lamina is reversibly depolymerized during mitosis. Cell, 19: 277287.

9. Goodpasture, C. and Bloom, S.E. 1975. Visualization of nucleolar organizer regions in mammalian chromosomes using silver staining. Chromosoma, 53: 37-50.

10. Hager, D.A. and Burgess, R.R. 1980. Elution of proteins from sodium dodecyl sulphate-polyacrylamide gels, removal of sodium dodecyl sulphate, and renaturation of enzymatic activity: results with sigma subunit of escherichia coli RNA polymerase, wheat germ DNA topoisomerase, and other enzymes. Anal. Biochem., 109: 76-86.

11. Harlow, E. and Lane, D. 1988. Antibodies. A Laboratory Manual. Cold Spring Harbour laboratory.

12. Hawkes, R., NidAy, E., and Gordon, J. 1982. A dot-immunobinding assay for monoclonal and other antibodies. Anal. Biochem., 119: 142-147.

13. Kaufmann, S.H. 1989. Additional members of the rat liver lamin polypeptide family. Structural and immunological characterization. J. Biol. Chem., 264: 13946-13955.

14. Klein, F., Laroche, T., Cardenas, M.E., Hofmann, J.F.-X., and SCHWEIZER, D. 1992. Localization of RAP 1 and topoisomerase II in nuclei and meiotic chromosomes of yeast. J. Cell. Biol., 117: 935-948.

15. Krohne, G. and Benavente, R. 1986. The nuclear lamins. A multigene family of proteins in evolution and differentiation. Exp. Cell. Res., 162: 1-10.

16. LAEmmLi, U.K. 1970. Cleavage of structural proteins during the assembly of the head of bacteriophage T4. Nature, 227: 680-685.

17. Lehner, C.F., Kurer, V., Eppenberger, H.M., and NigG, E.A. 1986. The nuclear lamin protein family in higher vertebrates: Identification of quantitatively minor lamin proteins by monoclonal antibodies. J. Biol. Chem., 261: 13293-13301.

18. Lowry, O.H., Rosebrough, N.J., FARR, A.L., and Randall, R.J. 1951. Protein measurement with the folin phenol reagent. J. Biol. Chem., 193: 265-275.

19. Merril, C.R., Goldman, D., Sedman, S.A., and Ebert, M.H. 1981. Ultrasensitive stain for proteins in polyacrylamide gels shows regional variation in cerebrospinal fluid proteins. Science, 211: 1437-1438.

20. Mura, C.V. and Stollar, B.D. 1981. Serological detection of homologies of $\mathrm{H} 1$ with $\mathrm{H} 5$ and $\mathrm{H} 1$ histones. J. Biol. Chem., 256: 9767-9769.

21. Platz, R.D., Meistrich, M.L., and Grimes, S.R. 1977. Low molecular weight basic proteins in spermatids. Methods in Cell Biol., XVI (297).

22. RaO, M.R.S., RAO, B.J., and Ganguly, J. 1982. Localization of testis variant histones on rat tesis chromatin. Biochem. J., 205: 15-21. 
23. RAYMOND, Y. and GAGNON, G. 1988. Lamin B shares a number of distinct epitopes with lamins $A$ and $C$ with intermediate filament proteins. Biochem., 27: 2597-2603.

24. SinGH, J. and RAO, M.R.S. 1987. Chromatin organization of sonication-resistant spermatid nuclei of rat testes. Ind. J. Biochem. Biophys., 24: 181-188.

25. Stewart, C. and Burke, B. 1987 . Teratocarcinoma stem cells and early mouse embryos contain only a single major lamin polypeptide closely resembling lamin B. Cell, 51: 383-392.

26. Stick, R. and Schwarz, H. 1982. The disappearance of the nuclear lamina during spermatogenesis: An electron microscopic and immunofluorescence study. Cell Differ., 11: 235-243.

27. Sudhakar, L. and RaO, M.R.S. 1990. Stage specific changes in the localization of a germ cell specific lamin during mammalian spermatogenesis. J. Biol. Chem., 265: 22526-22532.

28. SudhaKar, L., Sivakumar, N., Behal, A., and Rao, M.R.S. 1992. Evolutionary conservation of a germ cell specific lamin persisting through mammalian spermiogenesis. Exp. Cell. Res., 198: $78-84$.
29. Towbin, H., Staehelin, T., and Gordon, J. 1979. Electrophoretic transfer of proteins from polyacrylamide gels to nitrocellulose sheets: Procedure and some applications. Proc. Natl. Acad. Sci. USA, 76: 4350-4354.

30. Vester, B., Smith, A., Krohne, G., and Benavente, R. 1993. Presence of a nuclear lamina in pachytene spermatocytes of the rat. J. Cell. Sci., 104: 557-563.

31. von Wettstein, D., Rasmussen, S.W., and Holm, P.B. 1984. The synaptonemal complex in genetic segregation. Ann. Rev. Genet., 18: 331-431.

32. Westerwoudt, R.J., NaIPal, A.M., and Harrison, C.M.H. 1984. Improved fusion technique. II stability and purity of hybrid clones. J. Immunol. Meth., 68: 89-101.

(Received for publication, March 11, 1994

and in revised form, May 12, 1994) 